\title{
New Predictor of Atherosclerosis in Subjects With COPD: Atherogenic Indices
}

\author{
Sibel Gunay MD, Muzaffer Sariaydin MD, and Akif Acay MD
}

\begin{abstract}
BACKGROUND: We aimed to investigate the predictor role of the plasma levels of lipid parameters and atherogenic indices on development of atherosclerosis in subjects with COPD. METHODS: We retrospectively analyzed 104 male subjects diagnosed with stable COPD in hospital records. We excluded subjects with exacerbation, with known cardiovascular diseases and other chronic diseases, receiving anti-hyperlipidemic treatment, without sufficient past medical history, and lacking needed laboratory data. Additionally, 40 age-matched male healthy controls were also enrolled. C-reactive protein, total cholesterol, triglycerides, low-density lipoprotein cholesterol, and highdensity lipoprotein cholesterol levels of COPD and the control group were analyzed. Atherogenic indices (atherogenic index of plasma, cardiogenic risk ratio, and atherogenic coefficient) were calculated. RESULTS: C-reactive protein, triglyceride, atherogenic index of plasma, cardiogenic risk ratio, and atherogenic coefficient values were significantly higher in subjects with stable COPD than in control subjects $(P<.05$ for all). High-density lipoprotein cholesterol level was significantly lower in subjects with COPD than in the control group $(P<.001)$. C-reactive protein, lipid profiles, and atherogenic indices were similar in lower-risk (stage $A$ and $B$ ) and higher-risk (stage $C$ and $D$ ) subjects with COPD. Cardiogenic risk ratio and atherogenic coefficient were negatively correlated with $\mathrm{FEV}_{1}$ in all stable subjects with COPD and in higher-risk subjects with COPD $(r=-0.27$, $P=.01$ and $\mathrm{r}=-0.35, P=.01$, respectively). CONCLUSIONS: We concluded that atherogenic indices (atherogenic index of plasma, cardiogenic risk ratio, atherogenic coefficient) could be considered as a useful predictor for atherosclerosis and cardiovascular diseases in stable COPD patients. Nevertheless, further prospective investigations on this issue are warranted. Key words: atherogenic indices; atherosclerosis; cardiovascular diseases; COPD; comorbidity; lipid profile. [Respir Care 2016;61(11):1481-1487. () 2016 Daedalus Enterprises]
\end{abstract}

\section{Introduction}

COPD is a common preventable and treatable chronic respiratory disease that is characterized by exertional dys-

Dr Gunay is affiliated with the Chest Disease Clinic, Afyon State Hospital, Afyonkarahisar, Turkey. Dr Sariaydin is affiliated with the Department of Chest Diseases, Afyon Kocatepe University School of Medicine, Afyonkarahisar, Turkey. Dr Acay is affiliated with the Department of Internal Medicine, Afyon Kocatepe University School of Medicine, Afyonkarahisar, Turkey.

The authors have disclosed no conflicts of interest.

Correspondence: Sibel Gunay MD, Afyon Devlet Hastanesi, Gogus Hastaliklari Klinigi, 03200 Afyonkarahisar, Turkey. E-mail: sibelgunay@gmail.com.

DOI: $10.4187 /$ respcare.04796 pnea, wheeze, chronic cough, and expectoration resulting from progressive and partially reversible air-flow limitation associated with abnormal chronic inflammatory response of the lungs to noxious particles or gases. COPD is one of the leading causes of death and morbidity worldwide. ${ }^{1}$ During the course of the disease, systemic consequences and some concomitant diseases (such as cardiovascular diseases, skeletal muscle dysfunction, bronchial malignities, metabolic syndrome, diabetes mellitus, bronchiectasis, infections, impaired cognitive function, and depression) coexist. The relationships between cardiovascular diseases (including atherosclerosis, coronary artery diseases, and hypertension) and COPD were investigated in the previous studies. ${ }^{2-5}$ The main explanation for why patients with COPD more frequently suffer from cardiovascular diseases is the common systemic inflammatory pathway in the pathogenesis of both diseases. ${ }^{4,6}$ Furthermore, 
systemic low-grade chronic inflammatory processes and oxidative stress promote the development of both atherosclerosis and COPD. ${ }^{3,4}$ Atherosclerosis is the major contributor to cardiovascular diseases and the leading cause of global morbidity and mortality. Several algorithms have been established for early prediction of the presence of cardiovascular risk factors or atherosclerosis in the literature. . $^{72}$ For these prediction algorithms, several lipoprotein ratios or atherogenic indices (atherogenic index of plasma, cardiogenic risk ratio, and atherogenic coefficient) have been defined and investigated in several diseases.7,9,13,14 To the best of our knowledge, there is no study investigating the association between atherogenic indices in subjects with COPD in the current literature. For this reason, in the present study, we aimed to investigate the plasma levels of lipid parameters and calculated atherogenic indices in subjects with COPD as a predictor of atherosclerosis.

\section{Methods}

\section{Subjects With COPD and Control Subjects}

After obtaining local ethical committee approval and permission from our hospital administration, this study was conducted in the Chest Diseases Clinic, Afyon State Hospital, between September 2014 and August 2015.

COPD was diagnosed according to the Global Initiative for Chronic Obstructive Lung Disease (GOLD) definition. ${ }^{1}$ We retrospectively searched the hospital records for all patients who were admitted to the in-patient and out-patient chest disease clinic and diagnosed with COPD $(n=838)$. Hospital records were analyzed according to International Classification of Diseases, Tenth Revision (ICD-10) codes of COPD: J44, J44.0, J44.1, J44.8, and J44.9. Finally, we enrolled only $104(12.41 \%)$ male subjects with stable COPD (J44.8 and J44.9) for whom complete clinical data, including past medical history, exacerbation status for $1 \mathrm{y}$, dyspnea perceptions evaluated with Modified Medical Research Council and laboratory records (C-reactive protein and lipid profile, including the total cholesterol, triglyceride, high density lipoprotein cholesterol, and low density lipoprotein cholesterol), and pulmonary function tests, could be accessed. We searched all subjects' recordings, including previous drug use (such as anti-hyperlipidemic agents) on the online website of social insurance system for doctors of Turkey (https://medeczane.sgk.gov.tr/doktor/) by entering the identification numbers of subjects. Thus, we included stable COPD subjects who were not receiving treatment for hyperlipidemia. Additionally, we defined stable COPD according to the definition of Celli et al ${ }^{15}$ as stable symptoms with no further need for additional medications or doses of routine inhaler treatment for the last 3 months. Additionally, all subjects with COPD were categorized in groups A-D according to

\section{QUICK LOOK}

\section{Current knowledge}

During the course of COPD, systemic consequences and some comorbid diseases, including atherogenic cardiovascular diseases, coexist. Atherosclerosis is the major leading cause of morbidity and mortality worldwide. Additionally, systemic low-grade chronic inflammation and oxidative stress are common pathways that promote development of both COPD and atherosclerosis. Several algorithms have been established for early prediction of the presence of cardiovascular risk factors or atherosclerosis in the literature.

\section{What this paper contributes to our knowledge}

Our findings suggest that several lipoprotein ratios or atherogenic indices (atherogenic index of plasma, cardiogenic risk ratio, and atherogenic coefficient) could be considered as an easy and useful predictor for the development of atherosclerosis and cardiovascular diseases in stable COPD patients.

combined COPD risk assessment categories defined by GOLD (by using exacerbation risk [airway obstruction level according to $\mathrm{FEV}_{1}$, exacerbation, and hospitalization status within the previous $1 \mathrm{y}$ ] and dyspnea score [Modified Medical Research Council dyspnea score]). ${ }^{1}$

Our exclusion criteria were as follows: female sex, insufficient data in past medical history and laboratory data in hospital records, and the presence of exacerbation of COPD (defined according to Celli et al ${ }^{15}$ ) (ICD-10 codes $\mathrm{J} 44.0$ and J44.1), use of antihyperlipidemic agents, history of cardiovascular disease, systemic hypertension, renal failure, chronic liver disease, cerebrovascular events, diabetes mellitus, obstructive sleep apnea, and inflammatory bowel disease. Forty age-matched control subjects without any past medical history of chronic diseases or any other risk factors or who were referred to our out-patient chest disease clinics for their routine check-up were included.

\section{Pulmonary Function Test and Body Mass Index}

Pulmonary function tests were conducted $\geq 3$ times using the portable Spirolab III spirometer (Medical International Research, Waukesha, Wisconsin) in the pulmonary function test laboratory for all control subjects and all stable COPD subjects. The ratios of measured value to percent-of-predicted $\mathrm{FEV}_{1}$, percent-of-predicted FVC, and $\mathrm{FEV}_{1} / \mathrm{FVC}$ were noted. Weight $(\mathrm{kg})$ and height $(\mathrm{cm})$ of the subjects written in the pulmonary function test report was noted. Body mass index $\left(\mathrm{kg} / \mathrm{m}^{2}\right)$ was calculated as follows: body mass index $=$ weight $(\mathrm{kg}) /(\text { height }[\mathrm{m}])^{2}$. 
Table 1. Demographic Data of All Subjects in Both COPD and Control Groups

\begin{tabular}{|c|c|c|c|}
\hline Parameters & $\operatorname{COPD}(n=104)$ & Control $(n=40)$ & $P$ \\
\hline Age, mean $\pm \mathrm{SD}$ y & $64.0 \pm 8.7$ & $62.8 \pm 8.9$ & $.43 *$ \\
\hline $\mathrm{BMI}$, mean $\pm \mathrm{SD} \mathrm{kg} / \mathrm{m}^{2}$ & $24.72 \pm 3.91$ & $25.08 \pm 2.66$ & $.59 *$ \\
\hline \multicolumn{4}{|l|}{ Smoking status, $n(\%)$} \\
\hline Current smoker & $50(48.1)$ & $19(47.5)$ & \multirow[t]{3}{*}{$.34 \dagger$} \\
\hline Ex-smoker & $50(48.1)$ & $17(42.5)$ & \\
\hline Non-smoker & $4(3.8)$ & $4(10.0)$ & \\
\hline Smoking, mean \pm SD pack-years & $44.27 \pm 11.38$ & $35.76 \pm 12.88$ & $<.001 \ddagger$ \\
\hline \multicolumn{4}{|l|}{ Pulmonary function test, median (IQR) } \\
\hline FVC, $\%$ predicted & $66.00(29.50)$ & $80.00(16.50)$ & $<.001+$ \\
\hline $\mathrm{FEV}_{1}, \%$ predicted & $47.5(25.75)$ & $82.00(23.50)$ & $<.001 \ddagger$ \\
\hline $\mathrm{FEV}_{1} / \mathrm{FVC}, \%$ predicted & $61.00(8.00)$ & $84.50(11.75)$ & $<.001 \ddagger$ \\
\hline MMRC score & $2.00(2.00)$ & $1.00(1.00)$ & $<.001+$ \\
\hline Exacerbations during previous year, median (range) & $1(0-6)$ & NA & NA \\
\hline \multicolumn{4}{|l|}{ COPD stages, $n(\%)$} \\
\hline Stage A & $25(24)$ & NA & NA \\
\hline Stage B & $19(18.3)$ & NA & NA \\
\hline Stage C & $19(18.3)$ & NA & NA \\
\hline Stage D & $41(39.4)$ & NA & NA \\
\hline Risk category, $n(\%)$ & & & NA \\
\hline Higher risk (groups A and B) & $44(42.3)$ & NA & NA \\
\hline Lower risk (groups C and D) & $60(57.7)$ & NA & NA \\
\hline \multicolumn{4}{|l|}{$\begin{array}{l}\text { * Student } t \text { test. } \\
\dagger \text { Chi-square test. } \\
\text { ¥ Mann-Whitney U test. } \\
\text { BMI = body mass index } \\
\text { IQR = interquartile range } \\
\text { MMRC score = Modified Medical Research Council dyspnea score } \\
\text { NA = not applicable }\end{array}$} \\
\hline
\end{tabular}

\section{Laboratory Analysis}

Laboratory data of serum C-reactive protein level and lipid profile (total cholesterol, high-density lipoprotein cholesterol, low-density lipoprotein cholesterol, and triglyceride) were obtained from hospital records of subjects with stable COPD and control subjects. Atherogenic indices were calculated by using the value of lipid profile parameters according to the following formulas: atherogenic index for plasma $=\log$ (triglyceride/high-density lipoprotein cholesterol), where the concentrations of triglyceride and high-density lipoprotein cholesterol are in $\mathrm{mmol} / \mathrm{L}$, and calculation of the atherogenic index of plasma was performed using an online calculator ${ }^{16}$; cardiogenic risk ratio $=$ total cholesterol/high-density lipoprotein cholesterol; and atherogenic coefficient $=$ (total cholesterol high-density lipoprotein cholesterol)/high-density lipoprotein cholesterol.

\section{Statistical Analysis}

SPSS 20.0 for Windows (SPSS, Chicago, Illinois) was used to perform all statistical analysis. The distribution status of categorical variables was analyzed by the Kolmogorov-Smirnov test. Categorical variables were specified as $n(\%)$. All continuous variables were specified as mean \pm SD when normally distributed or median (interquartile range) when not normally distributed. Proportions in the different groups were compared by using the chi-square test. The Student $t$ test or Mann-Whitney U test was used to compare 2 independent groups according to distribution state. The Spearman's correlation test was used to analyze the correlation coefficients and their significance. A multiple linear regression model was used to determine independent predictors of all atherogenic indices. $P$ values $<.05$ were set to show a statistically significant result.

\section{Results}

The characteristics, pulmonary function test results of participants in both groups (stable COPD, $n=104$; healthy controls, $n=40$ ), and annual exacerbation status for the last $1 \mathrm{y}$ and COPD stages of the COPD group are outlined in Table 1. Although smoking status were statistically similar in both groups $(P=.34)$, cigarette consumption was higher in subjects with stable COPD than in control subjects 
Table 2. Lipid Profile and Atherogenic Indices of COPD and Control Subjects

\begin{tabular}{|c|c|c|c|}
\hline Parameters & $\operatorname{COPD}(n=104)$ & Control $(n=40)$ & $P$ \\
\hline C-reactive protein, median (IQR) $\mathrm{mg} / \mathrm{dL}$ & $0.95(0.63)$ & $0.40(0.46)$ & $<.001 *$ \\
\hline Total cholesterol, mean \pm SD mg/dL & $176.90 \pm 42.09$ & $177.59 \pm 42.66$ & $.93 \dagger$ \\
\hline HDL cholesterol, mean $\pm \mathrm{SD} \mathrm{mg} / \mathrm{dL}$ & $40.19 \pm 6.92$ & $45.82 \pm 7.42$ & $<.001 \dagger$ \\
\hline LDL cholesterol, mean $\pm \mathrm{SD} \mathrm{mg} / \mathrm{dL}$ & $103.73 \pm 34.88$ & $107.33 \pm 34.79$ & $.58 \dagger$ \\
\hline Triglycerides, mean $\pm \mathrm{SD}$ mg/dL & $163.43 \pm 46.56$ & $137.29 \pm 34.69$ & $.002 \dagger$ \\
\hline $\mathrm{AIP}$, mean $\pm \mathrm{SD}$ & $0.23 \pm 0.13$ & $0.11 \pm 0.11$ & $<.001 \dagger$ \\
\hline $\mathrm{CRR}$, mean $\pm \mathrm{SD}$ & $4.44 \pm 0.94$ & $3.91 \pm 0.92$ & $.002 \dagger$ \\
\hline $\mathrm{AC}$, mean $\pm \mathrm{SD}$ & $3.44 \pm 0.94$ & $2.91 \pm 0.92$ & $.002 \dagger$ \\
\hline $\begin{array}{l}\text { * Mann-Whitney } \mathrm{U} \text { test. } \\
\dagger \text { Student } t \text { test. } \\
\mathrm{IQR}=\text { interquartile range } \\
\mathrm{HDL}=\text { high-density lipoprotein } \\
\mathrm{LDL}=\text { low-density lipoprotein } \\
\mathrm{AIP}=\text { atherogenic indices in plasma } \\
\mathrm{CRR}=\text { cardiogenic risk ratio } \\
\mathrm{AC}=\text { atherogenic coefficient }\end{array}$ & & & \\
\hline
\end{tabular}

Table 3. Lipid Profile and Atherogenic Indices of Subjects in Higher- and Lower-Risk COPD Groups

\begin{tabular}{lccc}
\hline \hline \multicolumn{1}{c}{ Parameters } & Lower Risk $(n=44)$ & Higher Risk $(n=60)$ & $P$ \\
\hline C-reactive protein, median (IQR) mg/dL & $0.90(0.50)$ & $1.01(0.80)$ & $.15^{*}$ \\
Total cholesterol, mean $\pm \mathrm{SD} \mathrm{mg/dL}$ & $178.58 \pm 42.31$ & $.73 .68 \pm 43.24$ & $.73 \dagger$ \\
HDL cholesterol, mean $\pm \mathrm{SD} \mathrm{mg/dL}$ & $41.69 \pm 6.50$ & $.05 \pm 7.07$ \\
LDL cholesterol, mean $\pm \mathrm{SD} \mathrm{mg/dL}$ & $103.27 \pm 36.12$ & $104.06 \pm 34.25$ & $.91 \dagger$ \\
Triglycerides, mean \pm SD mg/dL & $165.86 \pm 43.95$ & $161.67 \pm 48.68$ & $.65 \dagger$ \\
AIP & $0.22 \pm 0.13$ & $0.24 \pm 0.12$ & $.63 \dagger$ \\
CRR & $4.35 \pm 1.09$ & $4.51 \pm 0.82$ & $.37 \dagger$ \\
AC & $3.35 \pm 1.09$ & $3.51 \pm 0.82$
\end{tabular}

$\overline{\text { Stages } \mathrm{A} \text { and } \mathrm{B} \text { are in }}$ the lower-risk group, and stages $\mathrm{C}$ and D are in the higher-risk group.

* Mann-Whitney U test.

$\dagger$ Student $t$ test.

$\mathrm{IQR}=$ interquartile range

HDL $=$ high-density lipoprotein

$\mathrm{LDL}=$ low-density lipoprotein

$\mathrm{AIP}=$ atherogenic indices in plasma

$\mathrm{CRR}=$ cardiogenic risk ratio

$\mathrm{AC}=$ atherogenic coefficient

(44.27 \pm 11.38 and $35.76 \pm 12.88$, respectively, $P<.001)$. Modified Medical Research Council dyspnea status and stages of stable COPD subjects are also shown in Table 1.

Serum C-reactive protein levels were significantly different in subjects with COPD and control subjects. Serum C-reactive protein levels and lipid profile of the 2 groups are outlined in Table 2. Serum high-density lipoprotein cholesterol level was significantly lower in subjects with stable COPD $(P<.001)$. Serum triglyceride level was significantly higher in subjects with stable COPD than in control subjects $(P=.002)$. Atherogenic indices (atherogenic index of plasma, atherogenic coefficient, and cardiogenic risk ratio) were significantly higher in subjects with COPD than in control subjects $(P<.001, P=.002$, and $P=.002$, respectively) (see Table 2).
The serum C-reactive protein levels, serum lipid profile, and atherogenic indices in subjects with lower risk (stage $\mathrm{A}$ and $\mathrm{B}$ COPD) and higher risk (stage $\mathrm{C}$ and $\mathrm{D}$ COPD) are outlined in Table 3. All parameters (C-reactive protein and lipid profile) measured in the serum of these 2 groups and all parameters calculated (atherogenic index of plasma, cardiogenic risk ratio, and atherogenic coefficient) were statistically similar in both groups (lower and higher risk COPD).

Correlation analysis showed that cardiogenic risk ratio and atherogenic coefficient were negatively correlated with $\mathrm{FEV}_{1}$ values in stable COPD subjects $(\mathrm{r}=-0.27, P=.01)$ (Table 4). Additionally, $\mathrm{FEV}_{1}$ value was correlated with cardiogenic risk ratio and atherogenic coefficient values in subjects with higher risk COPD $(\mathrm{r}=-0.35, P=.01)$ (Table 5). 


\section{ATHEROGENIC INDICES IN COPD}

Table 4. Correlation Analysis of Atherogenic Indices and Obstruction Level in Dynamic Pulmonary Function Test FEV $_{1}$ Results in Subjects With COPD and Control Subjects

\begin{tabular}{lccccc}
\hline \hline \multirow{2}{*}{$\begin{array}{l}\text { Atherogenic } \\
\text { Indices }\end{array}$} & \multicolumn{2}{c}{ COPD } & & \multicolumn{2}{c}{ Control } \\
\cline { 2 - 3 } \cline { 5 - 6 } & $\mathrm{r}$ & $P$ & & $\mathrm{r}$ & $P$ \\
\hline AIP & -0.04 & .70 & & -0.08 & .64 \\
CRR & -0.27 & .01 & & -0.01 & .94 \\
AC & -0.27 & .01 & & -0.01 & .94
\end{tabular}

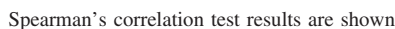

AIP $=$ atherogenic indices in plasma

$\mathrm{CRR}=$ cardiogenic risk ratio

$\mathrm{AC}=$ atherogenic coefficient

Table 5. Correlation Analysis of Atherogenic Indices and Obstruction Level in Dynamic Pulmonary Function Test FEV $_{1}$ Results in Higher- and Lower-Risk Subjects With COPD

\begin{tabular}{lcccccc}
\hline \hline \multirow{2}{*}{$\begin{array}{l}\text { Atherogenic } \\
\text { Indices }\end{array}$} & \multicolumn{2}{c}{ Lower Risk } & & \multicolumn{2}{c}{ Higher Risk } \\
\cline { 2 - 3 } \cline { 5 - 6 } & $\mathrm{r}$ & $P$ & & $\mathrm{r}$ & $P$ \\
\hline AIP & 0.03 & .86 & & 0.01 & .93 \\
CRR & -0.16 & .31 & & -0.35 & .01 \\
AC & -0.16 & .31 & & -0.35 & .01
\end{tabular}

Spearman's correlation test results are shown. Stages A and B are in the lower-risk group, and stages $\mathrm{C}$ and $\mathrm{D}$ are in the higher-risk group.

$\mathrm{AIP}=$ atherogenic indices in plasma

$\mathrm{CRR}=$ cardiogenic risk ratio

$\mathrm{AC}=$ atherogenic coefficient

Linear regression model for atherogenic index of plasma, cardiogenic risk ratio, and atherogenic coefficient were calculated as follows: atherogenic index of plasma $=0.15+(0.15 \times$ COPD diagnosis $*)+(-0.002 \times$ smoking consumption [pack-years]); cardiogenic risk ratio $=4.56+(0.32 \times \mathrm{COPD}$ diagnosis $*)+\left(-0.02 \times \mathrm{FEV}_{1}\right.$ $[\%$ predicted] $)$; atherogenic coefficient $=3.56+(0.32 \times$ COPD diagnosis $*)+\left(-0.01 \times \mathrm{FEV}_{1}[\%\right.$ predicted $\left.]\right)$ (*, COPD diagnosis value of 1 [if subject was diagnosed with COPD] or 0 [control subject, not diagnosed with COPD]).

\section{Discussion}

The first main outcome of the present study is that atherogenic lipid profiles and atherogenic indices (atherogenic index of plasma, atherogenic coefficient, and cardiogenic risk ratio), which were calculated from some ratios of serum cholesterol levels, were increased more in subjects with COPD than in healthy subjects. The other outcome is the correlation of these atherogenic indices with air-flow obstruction level $\left(\mathrm{eg}, \mathrm{FEV}_{1}\right.$ ) of subjects with higher-risk COPD.
COPD is a known major leading cause of morbidity and mortality worldwide. ${ }^{1}$ COPD frequently coexists with other diseases that may have an important role in disease prognosis. Cardiovascular diseases including heart failure, systemic hypertension, atrial fibrillation, and ischemic and atherosclerotic heart disease are a known major comorbidity in patients with COPD. ${ }^{1}$ In the literature, a strong association between airway obstruction in COPD and cardiovascular disease (especially ischemic heart disease) has been described. This association between COPD and cardiovascular disease suggests that patients with COPD might have a higher risk for cardiovascular disease, and patients with cardiovascular disease might have a higher risk for COPD. ${ }^{3-6,12,17}$ Systemic inflammatory response and its chronicity are the recommended common pathological pathway responsible for the development of these chronic diseases. ${ }^{4}$ Increased level of C-reactive protein, fibrinogen, and other inflammatory cytokines has been investigated in the development of both COPD and cardiovascular disease. Moreover, the relationship between reduced pulmonary function and carotid intima-media thickness is associated with atherosclerotic changes in subjects with COPD $^{4,18}$ Thus, these pathways might explain the development of concomitant chronic diseases in the same subject.4,6,18 Although it varies in some studies, the prevalence of ischemic heart diseases (including myocardial infarction, coronary stenosis, and angina pectoris) in subjects with COPD was reported to be between 4.7 and $60 \% .^{3,19}$ Furthermore, in a population-based study, Eriksson et al ${ }^{20}$ also showed a strong relationship between COPD and ischemic heart diseases. They reported that COPD was associated with ischemic heart disease (odds ratio 2.61) and ischemic heart disease with COPD (odds ratio 2.40).

Serum levels of C-reactive protein were reported as an inflammatory marker and indicator of disease activity in subjects with COPD. Additionally, higher C-reactive protein levels have also been found related to higher cardiovascular disease risk and atherogenesis. ${ }^{1,3,21-23}$ In this study, we also found a higher serum C-reactive protein level in subjects with COPD than in control subjects. Additionally, inflammation itself is associated with decreased serum highdensity lipoprotein cholesterol and increased triglyceride level. ${ }^{24}$ Hence, ongoing low grade inflammation in stable COPD may explain the common pathway with cardiovascular disease.

In the past decade, in addition to classical atherogenic risk factors, it was described that increases in some lipid ratios called atherogenic indices yield advantages in identifying some individuals with chronic diseases at higher risk of developing cardiovascular disease. ${ }^{7-15,25}$ To our knowledge, although atherogenic indices were studied to predict cardiovascular disease in some diseases, including diabetes mellitus, metabolic syndrome, systemic hypertension, familial Mediterranean fever, pregnancy-induced hy- 


\section{ATHEROGENIC INDICES IN COPD}

pertension, psychiatric diseases, and others, its role in COPD had not been studied. ${ }^{7-13,26,27}$ In the present study, we evaluated and compared atherogenic indices (atherogenic index of plasma, cardiogenic risk ratio, and atherogenic coefficient) in addition to the serum lipid parameters in subjects with COPD and in control subjects. We found that serum level of triglyceride and all atherogenic indices were significantly higher and serum high-density lipoprotein levels were significantly lower in subjects with stable COPD than in control subjects. These findings were parallel with the results of the previous studies. . $^{73,26,27} \mathrm{We}$ additionally found that these lipid levels and atherogenic indices did not differ in lower- or higher-risk groups for exacerbation.

Additionally, epidemiological data showed a strong relationship between air-flow limitation and cardiovascular disease. ${ }^{3,17}$ In this study, we also found a negative correlation between air-flow limitation levels $\left(\mathrm{FEV}_{1}\right)$ of COPD and both cardiogenic risk ratio and atherogenic coefficient.

In the literature, Arumalla et $\mathrm{al}^{28}$ studied the effect of the smoking status of subjects on cardiogenic risk ratio and atherogenic index of plasma. They reported a significant relationship between smoking status (smoker or nonsmoker) and atherogenic indices. In our study, smoking status was similar in COPD and control subjects. We found that the amount of cigarette consumption was significantly higher in the COPD group than in control subjects. Additionally, a multiple linear regression model for all atherogenic indices showed that smoking consumption amount is one of the independent predictors for the atherogenic index of plasma.

There are several limitations in the present study that should be specified. One is its retrospective design. Another important limitation is its cross-sectional design, which is not the best way to evaluate any causal relationship. Another limitation is the inclusion of only male participants. In this study, we excluded female patients because of the effect of menopause on estrogen levels and its effect on lipid profile of female subjects. ${ }^{29}$ Due to the retrospective design of the study, we excluded all female patients due to unknown menopausal status. We assumed that further prospective designed studies could overcome all of these limitations.

\section{Conclusions}

Novel atherogenic indices are warranted to predict atherogenic cardiovascular disease earlier in subjects with COPD. To the best of our knowledge, this is the first pilot study demonstrating the increased levels of atherogenic indices in subjects with COPD. Hence, we believe that atherogenic indices, including atherogenic index of plasma, cardiogenic risk ratio, and atherogenic coefficient, could be considered as a novel and useful tool to predict susceptibility to cardiovascular disease in subjects with COPD. However, further prospective studies with larger sample sizes are required to confirm and explore these results.

\section{REFERENCES}

1. The Global Initiative for Chronic Obstructive Lung Disease (GOLD). Global strategy for diagnosis, management, and prevention of chronic obstructive pulmonary disease. Updated 2016. http://goldcopd.org/ global-strategy-diagnosis-management-prevention-copd-2016/. Accessed March 9, 2016.

2. Fabbri LM, Luppi F, Beghé B, Rabe KF. Complex chronic comorbidities of COPD. Eur Respir J 2008;31(1):204-212.

3. Roversi S, Roversi P, Spadafora G, Rossi R, Fabbri LM. Coronary artery disease concomitant with chronic obstructive pulmonary disease. Eur J Clin Invest 2014;44(1):93-102.

4. Corbi G, Bianco A, Turchiarelli V, Cellurale M, Fatica F, Daniele A, et al. Potential mechanisms linking atherosclerosis and increased cardiovascular risk in COPD: focus on sirtuins. Int J Mol Sci 2013; 14(6):12696-12713.

5. Bellocchia M, Masoero M, Ciuffreda A, Croce S, Vaudano A, Torchio R, et al. Predictors of cardiovascular disease in asthma and chronic obstructive pulmonary disease. Multidiscip Respir Med 2013; 8(1):58.

6. Nussbaumer-Ochsner Y, Rabe KF. Systemic manifestations of COPD. Chest 2011;139(1):165-173

7. Acay A, Ulu MS, Ahsen A, Ozkececi G, Demir K, Ozuguz U, et al. Atherogenic index as a predictor of atherosclerosis in subjects with familial Mediterranean fever. Medicina 2014;50(6):329-333.

8. Caminhotto Rde O, da Fonseca FL, de Castro NC, Arantes JP, Sertié RA. Atkins diet program rapidly decreases atherogenic index of plasma in trained adapted overweight men. Arch Endocrinol Metab 2015;59(6):568-571.

9. Singh M, Pathak MS, Paul A. A study on atherogenic indices of pregnancy induced hypertension patients as compared to normal pregnant women. J Clin Diagn Res 2015;9(7):BC05-BC08.

10. Bakry OA, El Farargy SM, Ghanayem N, Soliman A. Atherogenic index of plasma in non-obese women with androgenetic alopecia. Int J Dermatol 2015;54(9):e339-e344.

11. Akbas EM, Timuroglu A, Ozcicek A, Ozcicek F, Demirtas L, Gungor A, Akbas N. Association of uric acid, atherogenic index of plasma and albuminuria in diabetes mellitus. Int J Clin Exp Med 2014;7(12):5737-5743.

12. Nunes SO, Piccoli de Melo LG, Pizzo de Castro MR, Barbosa DS, Vargas HO, Berk M, Maes M. Atherogenic index of plasma and atherogenic coefficient are increased in major depression and bipolar disorder, especially when comorbid with tobacco use disorder. J Affect Disord 2015;172:55-62.

13. Millán J, Pintó X, Muñoz A, Zúñiga M, Rubiés-Prat J, Pallardo LF, et al. Lipoprotein ratios: physiological significance and clinical usefulness in cardiovascular prevention. Vasc Health Risk Manag 2009; 5:757-765.

14. Essiarab F, Taki H, Lebrazi H, Sabri M, Saïle R. Usefulness of lipid ratios and atherogenic index of plasma in obese Moroccan women with or without metabolic syndrome. Ethn Dis 2014;24(2):207-212.

15. Celli BR, and MacNee W, ATS/ERS Task Force. Standards for the diagnosis and treatment of patients with COPD: a summary of the ATS/ERS position paper. Eur Respir J 2004;23(6):932-946.

16. Dobiášová M. Calculator of atherogenic risk. http://www.biomed. cas.cz/fgu/aip/calculator.php. Accessed February 1, 2016.

17. Schnell K, Weiss CO, Lee T, Krishnan JA, Leff B, Wolff JL, Boyd C. The prevalence of clinically-relevant comorbid conditions in pa- 


\section{ATHEROGENIC INDICES IN COPD}

tients with physician-diagnosed COPD: a cross-sectional study using data from NHANES 1999-2008. BMC Pulm Med 2012;12:26.

18. Iwamoto H, Yokoyama A, Kitahara Y, Ishikawa N, Haruta Y, Yamane $\mathrm{K}$, et al. Airflow limitation in smokers is associated with subclinical atherosclerosis. Am J Respir Crit Care Med 2009;179(1): $35-40$.

19. Müllerova H, Agusti A, Erqou S, Mapel DW. Cardiovascular comorbidity in chronic obstructive pulmonary disease: systematic literature review. Chest 2013;144(4):1163-1178.

20. Eriksson B, Lindberg A, Müllerova H, Rönmark E, Lundbäck B. Association of heart diseases with COPD and restrictive lung function: results from a population survey. Respir Med 2013;107(1):98-106.

21. Ridker PM, Bassuk SS, Toth PP. C-reactive protein and risk of cardiovascular disease: Evidence and clinical application. Curr Atheroscler Rep 2003;5(5):341-349.

22. Hancox RJ, Poulton R, Greene JM, Filsell S, McLachlan CR, Rasmussen F, et al. Systemic inflammation and lung function in young adults. Thorax 2007;62(12):1064-1068.

23. Günay E, Sarınç Ulaşlı S, Akar O, Ahsen A, Günay S, Koyuncu T, Unlü M. Neutrophil-to-lymphocyte ratio in chronic obstructive pulmonary disease: a retrospective study. Inflammation 2014;37(2):374380.
24. Esteve E, Ricart W, Fernández-Real JM. Dyslipidemia and inflammation: an evolutionary conserved mechanism. Clin Nutr 2005;24(1): 16-31.

25. Dobiásová M, Frohlich J. The plasma parameter log (TG/HDL-C) as an atherogenic index: correlation with lipoprotein particle size and esterification rate in apoB-lipoprotein-depleted plasma (FER(HDL)). Clin Biochem 2001;34(7):583-588.

26. Bortolasci CC, Vargas HO, Vargas Nunes SO, de Melo LG, de Castro MR, Moreira EG, et al. Factors influencing insulin resistance in relation to atherogenicity in mood disorders, the metabolic syndrome and tobacco use disorder. J Affect Disord 2015;179:148-155.

27. Tautu OF, Darabont R, Onciul S, Deaconu A, Comanescu I, Andrei $\mathrm{RD}$, et al. New cardiovascular risk factors and their use for an accurate cardiovascular risk assessment in hypertensive patients. Maedica 2014;9(2):127-134.

28. Arumalla VK, Vani N, Ramarao J. Dose-dependent impacts on the diagnostic efficacies of atherogenic lipids in adult Indian smokers. J Clin Diagn Res 2011;5(7):1352-1355.

29. Gaspard UJ, Gottal JM, van den Brûle FA. Postmenopausal changes of lipid and glucose metabolism: a review of their main aspects. Maturitas 1995;21(3):171-178. 